\title{
Analysis of a Flow Control System for a Combined Input-Crosspoint Buffered Packet Switch
}

\author{
Zhen Guo and Roberto Rojas-Cessa
}

\begin{abstract}
A flow control mechanism is used in combined input-crosspoint buffered (CICB) switches to avoid buffer overflow and underflow. Credit-based flow control is widely used in CICB switches to reduce the required crosspoint-buffer size. For simplification, we analyze an flow control mechanism with active queue management (AQM) and a proportional controller. We show that the flow control mechanism is stable for small crosspoint buffer sizes and non-negligible round-trip times. In addition, we observe the effect of an input shaper combined with a proportional controller. We show that the input shaper reduces the response time of the flow control mechanism.
\end{abstract}

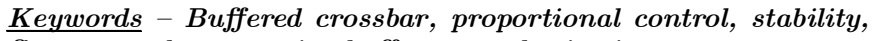
flow control, crosspoint buffer, round-trip time

\section{INTRODUCTION}

Combined input-crosspoint buffered (CICB) switches are an alternative to input-queued (IQ) packet switches to provide high-performance switching and to relax arbitration timing for packet switches with high-speed ports. CICB switches use time efficiently as input and output port selections are performed separately. CICB switches use virtual output queues (VOQs) at the inputs to avoid the head-of-line (HOL) problem, and internal transmission of fixed-length packets to simplify their implementation. Variable-size packets are segmented into cells at their arrival and reassembled at their departure from the switch.

A lot of research work on AQM mechanisms has been done recently. Most of this work focuses on supporting congestion management for the transmission control protocol (TCP) flows. The objective of AQM mechanisms is to provide early congestion notification to the sources so they can reduce the sending rate to avoid packet loss produced by buffer overflow [2].

We can consider a flow control mechanism where the parameter in observation is the queue occupancy. In this case, control theory can be applied to analyze and design queue management schemes. This paper focuses on applying control theory to analyze a flow control mechanism for a CICB switch.

\footnotetext{
*This work was supported in part by the National Science Foundation under Grants 0435250 and 0423305 , and in part by NJIT under Grant 421070.

The authors are with the Department of Electrical and Computer Engineering, New Jersey Institute of Technology, Newark, NJ 07102, USA. Email: \{zxg1554, rrojas\}@njit.edu.
}

Credit-based flow control is used for avoiding buffer overflow [1]. This flow control mechanism has been applied to CICB switches with a negligible transmission delay in switches [3], where the transmission delays are the propagation delays of sending a cell from the input port to a crosspoint, and the flow control information from the crosspoint buffer to the input port. The sum of the transmission delays plus the selection delays at inputs (VOQ selection) and outputs (crosspoint buffer selection) is called round-trip time. As the buffered crossbar switch can be physically located far from the input ports, actual round trip times $\left(R_{0}\right)$ can be non-negligible. Nonnegligible round trip delays have been considered in [4], [5], for practical implementations.

However, in a credit-based flow control mechanism, the stability of the switch, as a product of the round-trip time and crosspoint-buffer size, might be difficult to analyze. In this paper, we use a proportional $(\mathrm{P})$ controller for a flow control mechanism for a CICB switch. We analyze the relationship between the round-trip time and crosspoint buffer size and the effect on stability.

This paper is organized as follows. Section II briefly introduces the AQM-based flow control model in a CICB switch and presents the analysis on the relationship between the system stability and crosspoint buffer size as well as round-trip time. Section III discusses the design of an input shaper to obtain short transient response time of a flow control system. Section IV presents the conclusions.

\section{FLOW CONTROL MECHANISM AND STABILITY ANALYSIS}

Figure 1 shows a buffered crossbar switch with $M$ inputs and outputs. In this switch model, there are $M$ VOQs at each input. A VOQ at input $i$ that stores cells for output $j$ is denoted as $V O Q_{i, j}$. A crosspoint element in the buffered crossbar that connects input port $i$, where $0 \leq i \leq M-1$, to output port $j$, where $0 \leq j \leq M-1$, is denoted as $X P_{i, j}$. The buffer at $X P_{i, j}$ is denoted as $X P B_{i, j}$, and it is considered of $k$-cell size, where $k \geq 1$.

We consider that in a CICB switch each VOQ and its corresponding $X P B$ comprise a closed loop as shown in Figure 1. In order to avoid overflow of $X P B_{i, j}$, feedback is needed to inform the $V O Q_{i, j}$ to control the sending data 


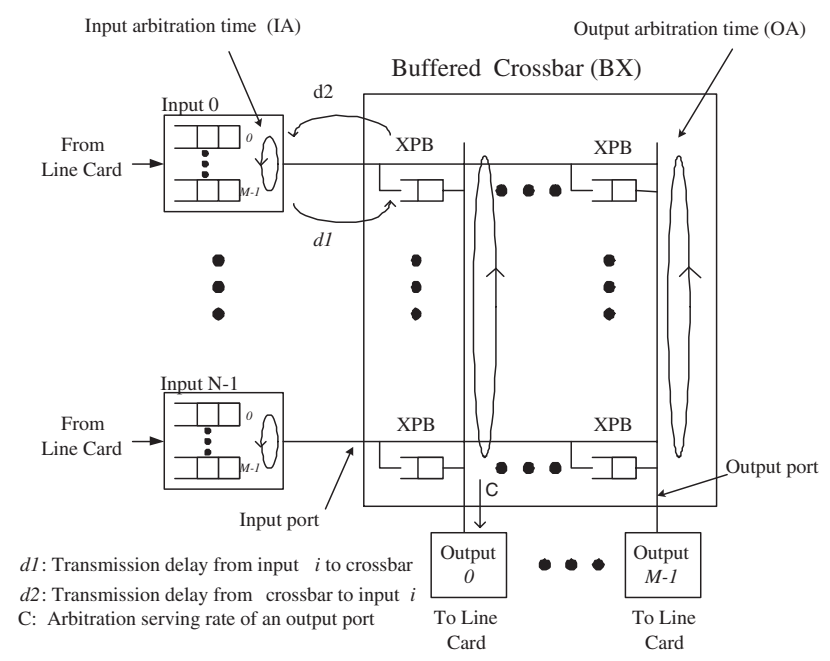

Fig. 1. Combine input-crosspoint buffered crossbar switch.

rate. Based on the similar concept of TCP windowing, we use a frame size to control the VOQ's sending rate. The frame size is the amount of packets transferring into the switch fabric [6],[7].

In TCP, the congestion window size, $W(t)$, is increased by one packet every time an acknowledgment is received in a round trip time if no congestion is detected, and is halved upon congestion detection. This additiveincrease multiplicative-decrease (AIMD) behavior of TCP has been modeled by the equation (1) and (2) in [2]:

$$
\dot{W}(t)=\frac{1}{R(t)}-\frac{W(t) W(t-R(t))}{2 R(t-R(t))} p(t-R(t)),
$$

where $W$ is the average TCP window size (packets), $R_{0}(t)$ is the round-trip time (secs).

In a network topology of $N$ homogeneous TCP sources and one router, the equation for the queue dynamics is given as:

$$
\dot{q}(t)=\frac{W(t)}{R(t)} N(t)-C
$$

where $q$ is the average queue length (packets), $C$ is link capacity (packets/sec) and $N$ is the load factor. After linearization of (1) and (2), we have the transfer function of the target plant:

$$
P(s)=\frac{\frac{C^{2}}{2 N}}{\left(s+\frac{2 N}{R_{0}^{2} C}\right)\left(s+\frac{1}{R_{0}}\right)} .
$$

To use this analogy in a CICB switch, some parameters need to be adapted. $W$ is the average VOQ frame size, $C$ is the arbitration serving rate of an output port (packets/sec). Since in each closed loop of $V O Q$ and its corresponding $X P B$ there is only one session, so the load factor is $N=1$. The minimum service rate to provide $100 \%$ throughput that one crosspoint buffer receives under uniform traffic is $\frac{C}{M}$, when $R_{0}$ can be negligible and $X P B$ size $k \geq 1$. In this paper, we consider a large $R_{0}$ value, where $\frac{k}{R_{0}}$ is not necessarily 1 . The minimum data rate of a flow that can be handled by this switch under uniform traffic is $\frac{C}{M} \frac{k}{R_{0}}$.

We discuss the feedback control on the following equation. The plant transfer function $P(s)$ is:

$$
P(s)=\frac{\frac{C^{2} k^{2}}{2 M^{2} R_{0}^{2}}}{\left(s+\frac{2 M}{R_{0} C k}\right)\left(s+\frac{1}{R_{0}}\right)} .
$$

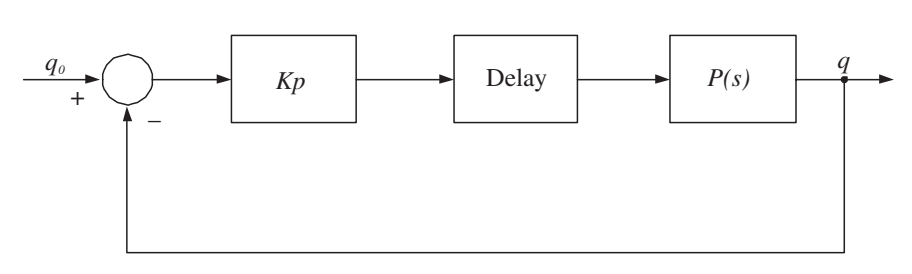

Fig. 2. Block Diagram of $\mathrm{P}$ control in a VOQ-XPB closed loop.

We use P control for the flow control in a CICB switch. Figure 2 shows the block diagram of the $\mathrm{P}$ control in the VOQ-XPB closed loop. The feedback signal is the regulated output (crosspoint buffer occupancy) multiplied by a gain factor $K_{p}$.

The nominal loop transfer function of the proportional controller case is:

$$
L(s)=\frac{K_{p} \frac{C^{2} k^{2}}{2 M^{2} R_{0}^{2}} e^{-s R_{0}}}{\left(s+\frac{2 M}{R_{0} C k}\right)\left(s+\frac{1}{R_{0}}\right)} .
$$

We can take the loop's unity-gain crossover frequency as the geometric mean of corner frequency:

$$
w_{g}=\sqrt{\frac{2 M}{R_{0}^{2} C k}}
$$

and choose $K_{p}$ to make $\left|L\left(j w_{g}\right)\right|=1$. The phase margin $(P M)^{1}$ is

\footnotetext{
${ }^{1}$ Phase margin, as a relative stability indicator for both discretetime and continuous time system, is defined in terms of the system open-loop frequency response [8].
} 
TABLE I

Phase Margin AND the RATIO OF CROSSPoint BUfFER SIZE AND ROUND-TRIP TIME

\begin{tabular}{||c|c|c||}
\hline \hline$k$ & $k / R_{0}$ & $\mathrm{PM}$ \\
\hline 10 & $\frac{1}{10}$ & $-54.85^{\circ}$ \\
20 & $\frac{1}{5}$ & $-12.43^{\circ}$ \\
25 & $\frac{1}{4}$ & $-1.62^{\circ}$ \\
30 & $\frac{1}{3}$ & $6.36^{\circ}$ \\
40 & $\frac{2}{5}$ & $17.59^{\circ}$ \\
50 & $\frac{1}{2}$ & $25.23^{\circ}$ \\
100 & 1 & $44.19^{\circ}$ \\
\hline \hline
\end{tabular}

$P M=180-\arctan w_{g} R_{0}-\arctan \frac{w_{g} R_{0} C k}{2 M}-\frac{180}{\pi} w_{g} R_{0}$.

Let's consider the following example.

Example 1: Consider the following setup in a CICB switch. $C=3750$ packets $/ \mathrm{s}, M=32, R_{0}=0.0246 \mathrm{~s}$, and the value of $k$ is the crosspoint buffer size in time slots.

Since $C=3750$ packets $/ \mathrm{s}$, one time slot is $0.267 \mathrm{~ms}$. For example, $k$ is 10 -packet long, or $k=2.67 \mathrm{~ms}$.

Assume that the round-trip time is fixed. When the crosspoint buffer size increases, the system's phase margin increases as Table I shows. However, when crosspoint buffer size decreases to a value where $\frac{k}{R_{0}} \leq \frac{1}{4}$, the system becomes unstable. ${ }^{2}$

To support large $R_{0}$ in a buffered crossbar switch, the crosspoint-buffer size need to be large enough, such that up to $R_{0}$ cells can be buffered. However, the memory amount that can be allocated in a chip is limited. In this example, when $\frac{k}{R_{0}} \geq \frac{1}{3}$, the flow control system is stable.

\section{DESIGN OF AN INPUT SHAPER}

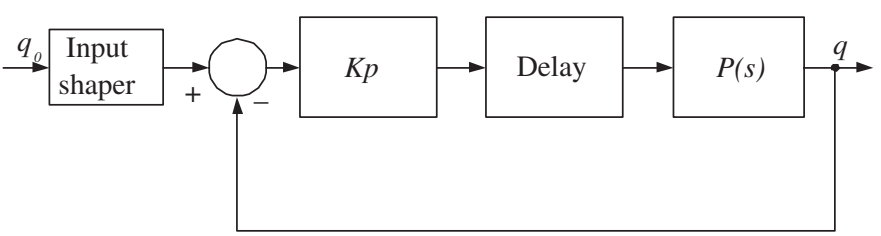

Fig. 3. Block Diagram of $\mathrm{P}$ control with input shaper in a VOQ-XPB closed loop.

For the purpose of the flow control in a CICB switch, the controller must have an acceptable transient response such that the crosspoint buffer occupancy can converge

${ }^{2}$ In most cases, a positive phase margin will ensure stability of the closed-loop system [8]. to a target value $q_{0}$ in a short time. A short transient response time is important to the flow control in a CICB switch.

In order to obtain a short transient response, we add an input shaper to the closed loop system. The input shaper is a feed-forward pole-zero cancelation method. Ideally, the input shaper uses its zeros to cancel the poles of a target system. Therefore, a good performance is obtained [11].

The input shaper might be designed as a step function. In our case, the step function is used to set up the value of $q_{0}$ and also the time to apply $q_{0}$ into the closed-loop system. Each step function has a step response. We use two step functions to cancel the two-pole system's overshoots. The key point is how to put these two step functions together. We show this combination with the following example.

Example 2: Consider the following setup in a CICB switch. $C=3750$ packets $/ \mathrm{s}, M=32, R_{0}=0.00801 \mathrm{~s}$, and the value of $k$ is $0.00267 \mathrm{~s}$, the ratio $\frac{k}{R_{0}}=1 / 3$.

The closed-loop system can be regarded as a second-order system by approximation [12]. The transient response is shown in Figure 5. Because the system is underdamped, there are some oscillations before the crosspoint buffer occupancy converges to $q_{0}$. The maximum overshoot value $q_{\max }$ is measured as $q_{\max }=15$, the steady state value of queue occupancy $q_{s s}$ is measured as $q_{s s}=10$.

With the following equations, we can obtain the step value and step time for each step function.

$$
V=\frac{q_{\max }-q_{s s}}{q_{s s}}=0.5
$$

The step value for the first step function is:

$$
A_{1}=\frac{1}{1+V}
$$

The step value for the second step function is:

$$
A_{2}=1-A_{1}
$$

$$
\exp \left(-\frac{\zeta \pi}{\sqrt{1-\zeta^{2}}}\right)=V
$$

From (11), the damping factor $\zeta$ is obtained as 0.2154 . Since $\zeta$ is less than 1 , the system is underdamped. Then, the time interval between the first step function and the second step function is obtained. 


$$
\Delta T=\frac{\pi}{\omega_{n} \sqrt{1-\zeta^{2}}}=0.01573
$$

In summary, if the target crosspoint buffer occupancy is $q_{0}$, with a design of an input shaper, we first generate a step function whose step value is $q_{0} \times A_{1}$, and its step time is 0 . The step value of the second step function is $q_{0} \times A_{2}$ and its step time is $\Delta T$. Now, we get the input shaper. Before being input to the proportional control system as Figure 2 shows, $q_{0}$ is shaped by the two step functions. That is why we call the two step functions an input shaper. Figure 4 shows the block diagram of a $\mathrm{P}$ control with input shaper. As Figures 5 and 6 show, with an input shaper, the transient response time can be improved by almost $40 \%$.

We show the simulation result for a Proportional-Integral (PI) control with input shaper and its comparison with the PI control without input shaper. Figure 7 shows the block diagram of a PI control with input shaper. As Figures 8 and 9 show, we can obtain the similar result as the above $\mathrm{P}$ control such that the transient response time can be improved by almost $40 \%$.

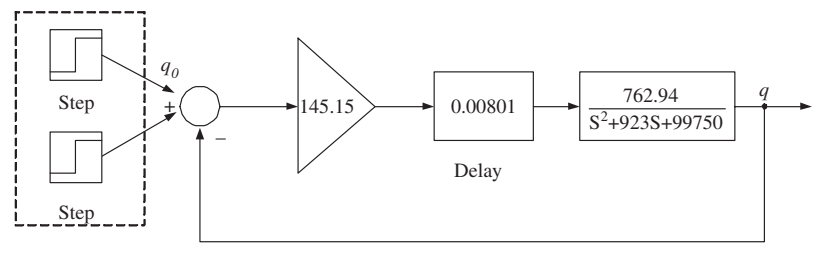

Input shaper

Fig. 4. Diagram of a $\mathrm{P}$ control with input shaper.

\section{CONCLUSIONS}

In this paper, we analyzed an AQM-based flow-control mechanism for CICB switches. We investigated the stability margin by analyzing the relationship between the crosspoint buffer size and the round-trip time. We show that when $\frac{k}{R_{0}} \geq \frac{1}{3}$ in our experiments, the flow control system is stable. This may provide a guideline on how to choose the crosspoint buffer size under non-negligible round trip times. Also, we improved the system's transient response time by almost $40 \%$ when adding an input shaper to the closed-loop system.

\section{References}

[1] H. T. Kung, K. Chang, "Receiver-Oriented Adaptive Buffer Allocation in Credit-Based Flow Control for ATM Networks," Proc. of IEEE INFOCOM'95, Vol. 1, pp. 239-252.

[2] C. V. Hollot, V. Misra, D. Towsley and W. Gong, "Analysis and Design of Controllers for AQM Routers Supporting TCP Flows," IEEE Transactions on Automatic Control, Vol. 47, No. 6 , June 2002, pp. 945-959

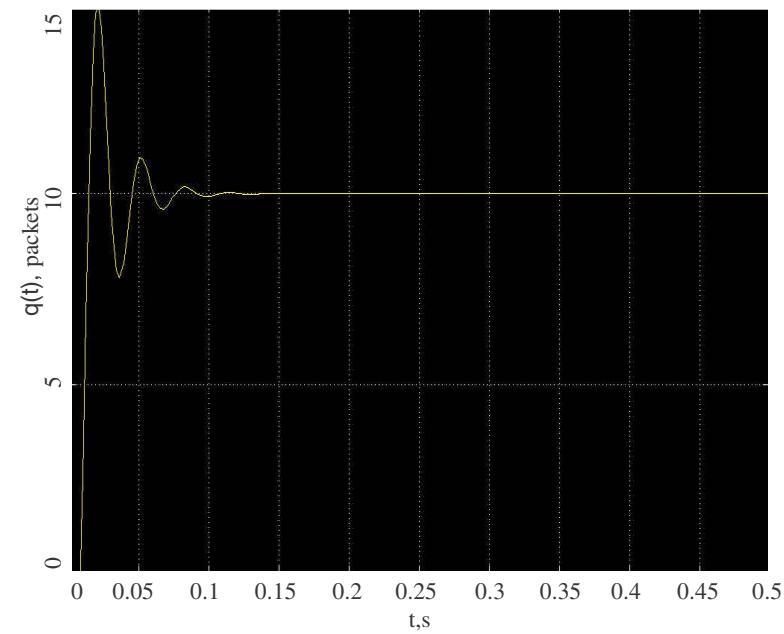

Fig. 5. Simulation result on a $\mathrm{P}$ control without input shaper.

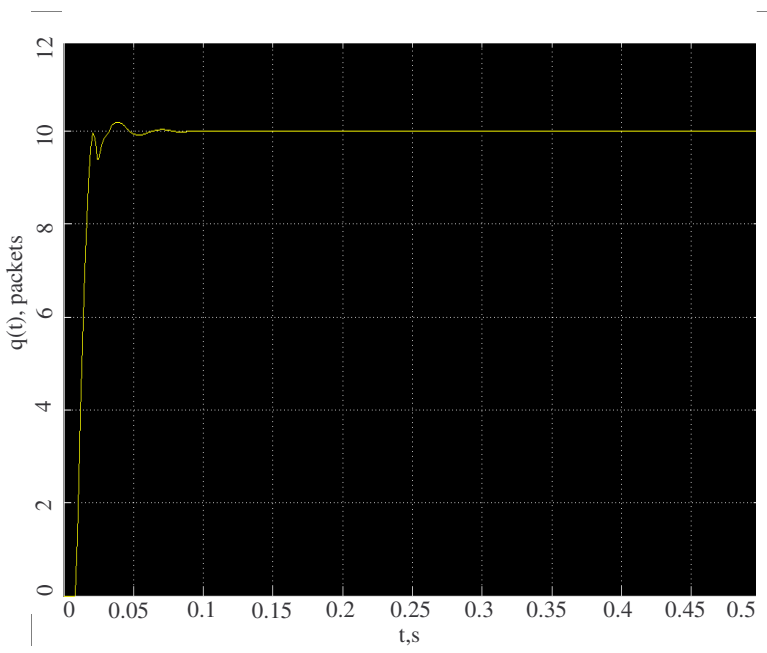

Fig. 6. Simulation result on a $\mathrm{P}$ control with input shaper.

[3] R. Rojas-Cessa, E. Oki, Z. Jing, and H. J. Chao "CIXB-1: Combined Input-One-Cell-Crosspoint Buffered Switch," Proc. of IEEE Workshop of High Performance Switches adn Routers 2001, pp. 324-329, May, 2001.

[4] R. Luijten, C. Minkenberg, and M. Gusat, "Reducing Memory Size in Buffered Crossbar with Large Internal Flow Control Latency," Proc. IEEE Global Telecommunications Conference 2003, Vol. 3, pp. 3683-3687.

[5] F. Gramsamer, M. Gusat, and R. Luijten, "Optimizing Flow Control for Buffered Switches," Proc. IEEE Proc. of International Conference on Computer Communications and Networks 2002, pp.438-443.

[6] A. Bianco, M. Franceschinis, et. al., "Frame-Based Matching Algorithms for Input-Queued Switches," Proc. IEEE High Performance Switches and Routers 2002, May 2002, pp. 69-76.

[7] R. Rojas-Cessa, E. Oki, "Round-robin Selection with 


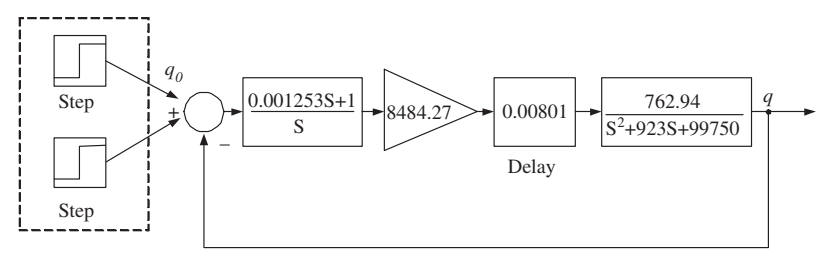

Input shaper

Fig. 7. Diagram of a PI control with input shaper.

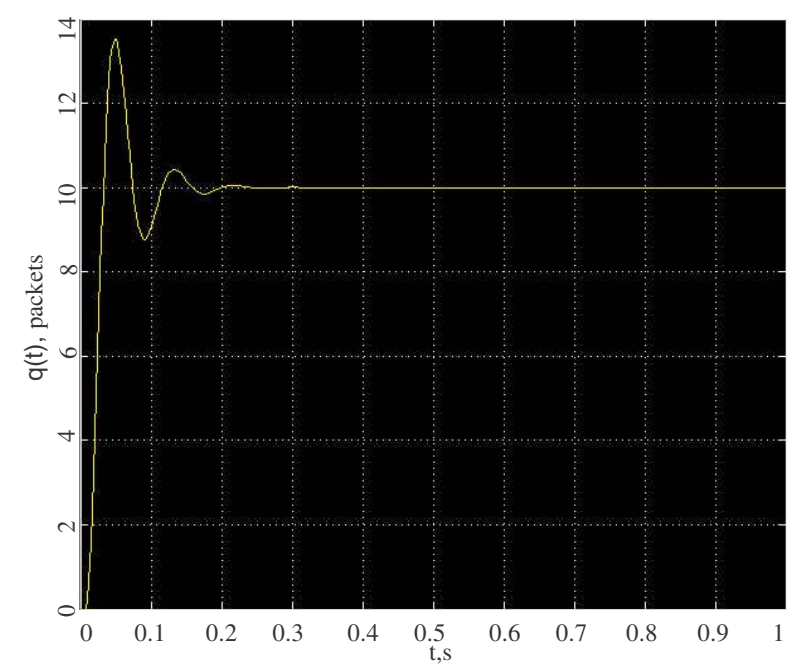

Fig. 8. Simulation result on a PI control without input shaper.

Adaptable-Size Frame in a Combined Input-Crosspoint Buffered Switch," IEEE Communications Letters, Vol. 7, No. 11, Nov., 2003, pp. 555-557.

[8] J. DiStefano, A. Stubberud and I. Williams, "Feedback and Control Systems," Schaum's Outline Series, McGRAW-HILL, Inc. 1990.

[9] S. Floyd, V. Jacobson, "Random Early Detection Gateways for Congestion Avoidance," IEEE/ACM Trans. Networking, Vol. 1, pp. 397-413, Aug. 1993.

[10] F. Ren, C. Lin, "Speed up the Responsiveness of Active Queue Management System," IEICE Trans. Commun., Vol. E86-B, No. 2, pp. 630-636, 2003.

[11] N. C. Singer, W. P. Seering, "Preshaping Command Inputs to Reduce System Vibrations," ASME Journal of Dynamic Systems, Measurement, and Control, Vol. 112, No. 1, pp. 7682, 1990 .

[12] Q. Chen, Q. W. W. Yang "AQM Controller Design for IP routers Supporting TCP Flows Based on Pole Placement," IEE Proc.-Commun., Vol. 151, No. 4, pp. 347-354, 2004.

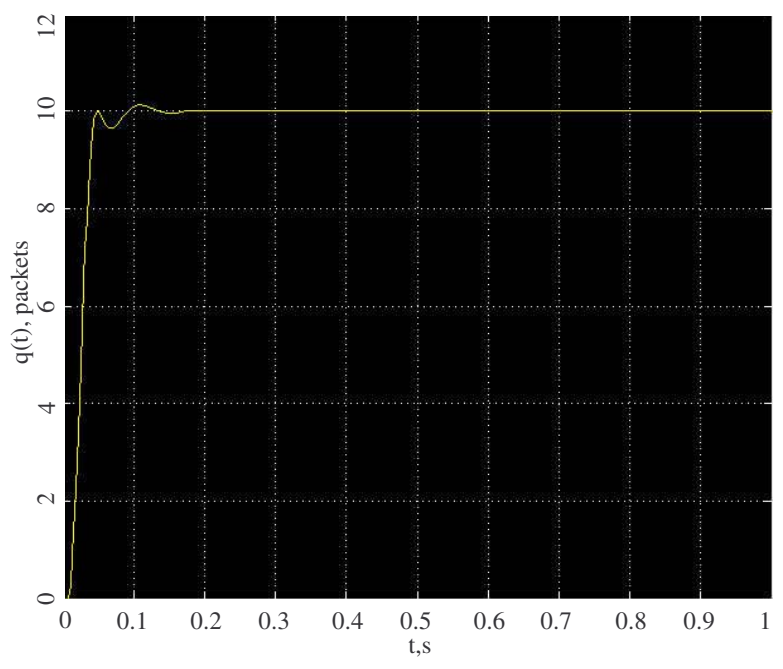

Fig. 9. Simulation result on a PI control with input shaper. 